\title{
Magnetic resonance imaging-based body composition is associated with nutritional and inflammatory status: a longitudinal study in patients with Crohn's disease
}

\author{
Ziling Zhou ${ }^{1,2 \dagger}$, Ziman Xiong ${ }^{1 \dagger}$, Yaqi Shen ${ }^{1 *}\left(\mathbb{0}\right.$, Zhen $^{1}{ }^{1}$, Xuemei $\mathrm{Hu}^{1}$ and Daoyu Hu${ }^{1}$
}

\begin{abstract}
Objective: To evaluate the changes in magnetic resonance imaging-based body composition parameters during follow-ups in patients with Crohn's disease (CD).

Methods: Between November 1, 2017, and June 30, 2021, patients diagnosed with CD, who underwent two or more magnetic resonance enterography (MRE) scans at our institution were retrospectively reviewed. The baseline and one subsequent follow-up scan for each patient were paired to form longitudinal comparisons. Skeletal muscle, visceral adipose tissue (VAT), and subcutaneous adipose tissue (SAT) indexes were calculated from tissue areas measured at the third lumbar vertebra level per scan, standardized by dividing the height ${ }^{2}$ and lumbar height ${ }^{2}$ (height L1-L5). We also assessed the correlation between changes in VAT to total adipose tissue ratio (VA/TA index) and CD activity scores (5-point MRE classification) using Spearman's correlation analysis. A multivariate linear regression model was used to adjust for the follow-up duration and treatment type.
\end{abstract}

Results: Overall, 49 patients (with 49 paired scans) were enrolled. VA/TA index changes were negatively correlated with changes in skeletal muscle index (SMl; $r=-0.339, p<0.05)$. The VA/TA index $(52.69 \pm 10.66 \%$ vs. $49.18 \pm 10.80 \%$, $p<0.001)$ and the total MRE score $(8.0 \pm 3.9$ vs. $5.7 \pm 3.4, p<0.001)$ decreased significantly during follow-up, regardless of follow-up duration and treatment type (both $p>0.05$ ). Changes in total MRE score were negatively correlated with SMI changes $(r=-0.408, p<0.01)$ but positively correlated with VA/TA index changes $(r=0.479, p<0.01)$.

Conclusion: An increase in SMI and a decrease in VA/TA index could reflect improved nutritional and inflammatory status.

Keywords: Crohn disease, Magnetic resonance enterography, Visceral adipose tissue, Drug therapy

\section{Key points}

1. MRI-based body composition parameters can reflect disease course in CD patients.

*Correspondence: yqshen@hust.edu.cn

†Ziling Zhou and Ziman Xiong contributed equally to this work and should be considered co-first authors.

${ }^{1}$ Department of Radiology, Tongji Hospital, Tongji Medical College, Huazhong University of Science and Technology, 1095 Jiefang Avenue,

Qiaokou District, Wuhan 430030, Hubei, China

Full list of author information is available at the end of the article

2. Decrease of VA/TA index correlated with improvement of disease activity during follow-up.

3. Higher SMI may indicate better nutritional status. 


\section{Background}

Crohn's disease (CD) is a chronic, inflammatory, gastrointestinal disease. According to previous studies, patients often suffer from malnutrition during the disease course [1]; thus, they face a higher risk of post-operative infections and a longer in-hospital stay [2]. Weight loss is an intuitive indication of malnutrition and has been considered one of the main features of $\mathrm{CD}$; it is often accompanied by sarcopenia, which is related to inflammation, reduced physical activity, and glucocorticoid use [1,3,4]. Generally, CD patients were found to gain weight after anti-TNF therapy [5]. Regardless of weight gain or loss, skeletal muscle, subcutaneous fat, and visceral fat exhibit different changes during the process of weight change; however, anthropometric measurements, including abdominal circumference and body mass index (BMI)-routinely used in clinical practice-do not provide an accurate assessment of body composition changes.

Cross-sectional imaging, including computed tomography $(\mathrm{CT})$ and magnetic resonance imaging (MRI), are widely used for body composition analysis [6-8]. We can obtain different body composition parameters by performing semi-automatic or manual outlining of different tissues on monolayer images at the L3 level. MRI-based quantitative analyses of muscle, visceral adipose tissue (VAT) and subcutaneous adipose tissue (SAT) have been proposed to be as reliable as CT in assessing body fat distribution and muscle content $[9$, 10]. Compared with $C T, M R$ has no ionizing radiation and has a high accuracy for detecting disease activity when applied for surveillance of CD patients [11]. A link was found between MRI-based body composition parameters and inflammatory status in CD patients. For example, Büning et al. [12] found a higher proportion of VAT in women with CD than control women; Labarthe et al. [13] also found a higher proportion of VAT in patients with active $C D$ compared to inactive patients. However, the former study group was all female, while the latter was a cross-sectional comparison of patients with different levels of activity, and few studies have assessed patients longitudinally by monitoring both changes in body composition and inflammatory status assessed by MRI during follow-up, that is, the association between changes of MRI-based body composition and changes of disease activity during treatment was still unclear.

We aimed to evaluate changes in the nutritional and inflammatory status of $\mathrm{CD}$ patients by comparing longitudinal clinical indicators, intestinal lesions, and MRbased body composition parameters during follow-up.

\section{Methods}

The local review board approved this retrospective study, and the requirement of informed consent was waived.

\section{Patients}

All patients who underwent MR enterography (MRE) scans at our institution between 1 November 2017 and 30 June 2021 were reviewed, and those with two scans and a diagnosis of $\mathrm{CD}$ were enrolled. The diagnosis was confirmed by an experienced inflammatory bowel disease multidisciplinary team at our institution according to the ECCO-ESGAR guidelines [14], combining information from endoscopy, biopsy, laboratory tests, imaging findings, and history review. Patients were further excluded if: (1) they underwent bowel resection between their first and subsequent scans; (2) clinical data on treatment information or laboratory information reflecting nutritional status between two scans was unavailable.

After identifying patients who met the above criteria, information (sex, age at inclusion, family history, smoking history) at the time of the first MRE per patient was recorded from the electronic medical record system. Clinical data (age, height, weight, BMI, specific CDrelated treatment, erythrocyte sedimentation rate (ESR), C-reactive protein (CRP), albumin (Alb), and haematocrit (Hct) levels) and the Montreal classification [15] were recorded within seven days of each MRE scan. BMI was obtained according to the formula $\mathrm{BMI}=$ body weight $(\mathrm{kg}) /$ height $^{2}\left(\mathrm{~m}^{2}\right)$ and was categorized as normal (18.524.9), underweight $(<18.5)$, and overweight (25-30) [16]. We paired each patient's initial scan with one subsequent follow-up scan to form a longitudinal comparison group.

\section{MRE examinations}

All examinations are performed on one of four 3.0 T MR scanners (GE Discovery 750HD, GE Healthcare; Siemens MAGNETOM Skyra (2 sets), Siemens AG; uMR 780, United Imaging Healthcare). All patients underwent pre-scan preparation as recommended by the European Society of Gastrointestinal and Abdominal Radiology (ESGAR) and European Society of Pediatric Radiology (ESPR) [17]; that is, fasting for $4-6 \mathrm{~h}$ before the scan and drinking $1000-1500 \mathrm{~mL}$ of $2.5 \%$ aqueous mannitol solution regularly within $30-45 \mathrm{~min}$ before the scan. To minimize bowel peristalsis, $10 \mathrm{mg}$ anisodamine was orally administered $30 \mathrm{~min}$ before the examination to patients without contraindications, including glaucoma and prostatic hypertrophy. Additionally, routine MRE sequences were consistent with those suggested by ESGAR/ESPR, as shown in the Additional file 1: Table S1. 


\section{Images analysis}

Digital imaging and communications in medicine images of the MRE sequence were extracted per patient for quantification of body composition parameters and assessment of inflammatory involvement of the intestine.

\section{Quantification of body composition parameters}

One research assistant extracted a single slice at the midlevel of the third lumbar vertebra (L3) from the axial T1-weighted images for further evaluation. Segmentation and measurement of skeletal muscle, SAT, and VAT were performed by a semi-automated method [18] using ImageJ (National Institutes of Health, https://imagej. nih.gov/ij/index.html), as shown in Fig. 1. Thereby, we obtained cross-sectional areas $\left(\mathrm{cm}^{2}\right)$ of skeletal muscle, SAT, and VAT. Additionally, we measured the height between the upper edge of the first and lower edge of the fifth lumbar vertebrae in each scan (height ${ }_{\mathrm{L} 1-\mathrm{L} 5}, \mathrm{~cm}$ ), which is illustrated in Fig. 1. The quantification processes were separately performed by two radiologists blinded to the patient's medical information; the average of the two measurements was used for subsequent analysis. Additionally, the skeletal muscle, SAT, and VAT areas were normalized by both height square $\left(\mathrm{m}^{2}\right)$ and height $\mathrm{L}_{\mathrm{L}-\mathrm{L} 5}$ square $\left(\mathrm{cm}^{2}\right)$ to obtain the skeletal muscle index (SMI and $\mathrm{SMI}_{\text {spinal }}$ ), subcutaneous adipose index (SAI and
$\left.\mathrm{SAI}_{\text {spinal }}\right)$, and visceral adipose index (VAI and $\left.\mathrm{VAI}_{\text {spinal }}\right)$, respectively. Sarcopenia was defined as: $\mathrm{SMI}<49.9 \mathrm{~cm}^{2} /$ $\mathrm{m}^{2}$ for men; and $\mathrm{SMI}<28.7 \mathrm{~cm}^{2} / \mathrm{m}^{2}$ for women, according to previously defined cut-off value [19]. We calculated the percentage of VAT to total adipose tissue (VA/TA index) in a single layer based on the formula VAT area/ $($ SAT area + VAT area $) \times 100 \%$.

\section{Qualitative assessment of the intestine}

We evaluated the intestinal lesions in each scan, referring to the 5-Point MR enterocolonography classification [20]. The procedure consists of visual scoring of the jejunum, distal ileum, terminal ileum, ascending colon, transverse colon, descending colon, sigmoid colon, and rectum, where there is: 0 : normal or no lesions; 1 : inactive or quiescent $\mathrm{CD}$; 2 : mild active inflammation; 3 : moderate active inflammation; 4: severe active inflammation. Two radiologists evaluated each scan, and any inconsistencies between them were reviewed by another senior radiologist, and a final decision was made accordingly. The MRE scores for the small bowel (jejunum, distal ileum, and terminal ileum) and colorectum (colon and rectum) were calculated separately and then added together to obtain the total MRE score for each scan. According to the MRE score criteria proposed by Kitazume et al., we defined intestinal improvement as: a reduction in total
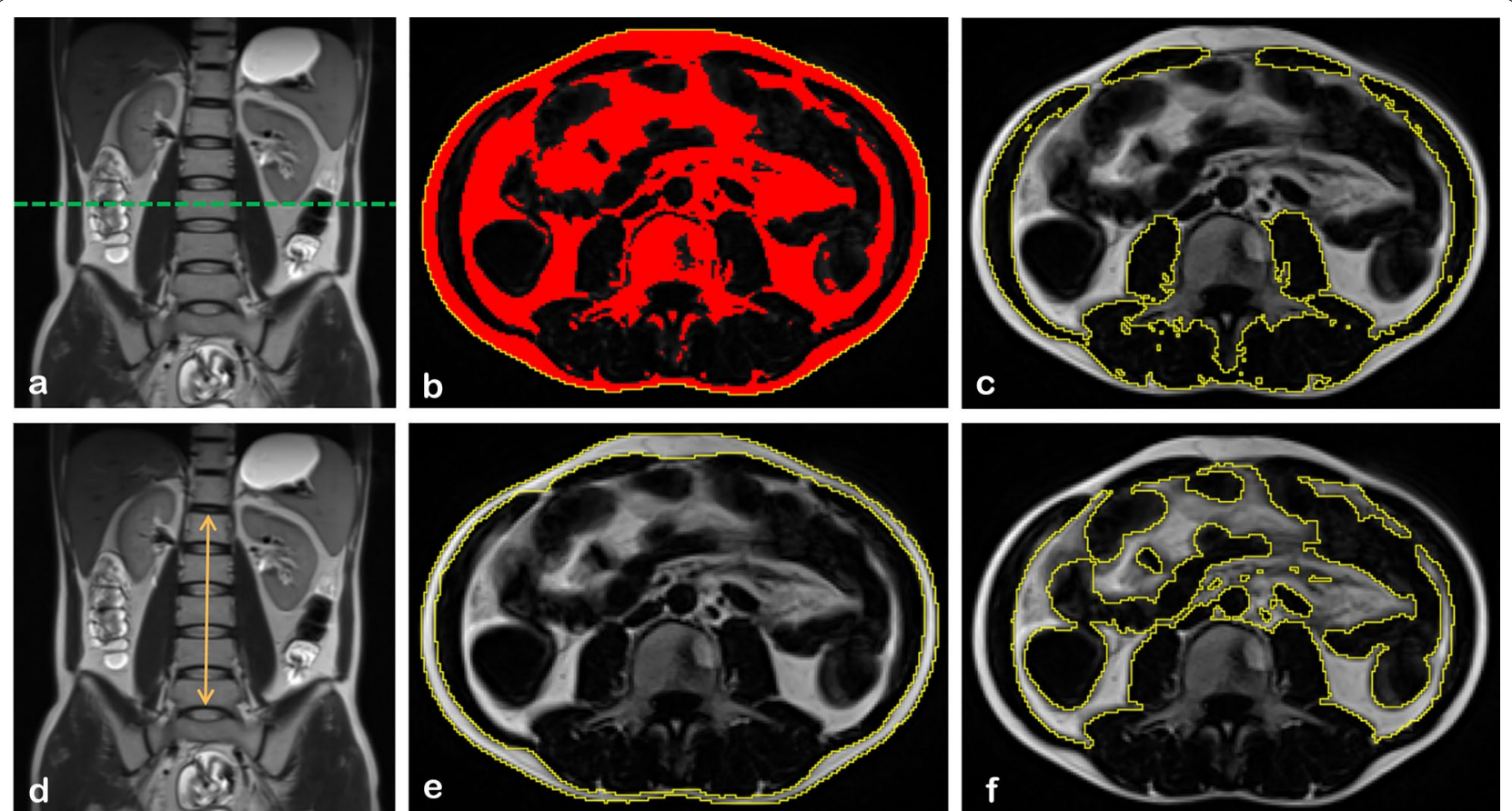

Fig. 1 Extraction diagram of body composition parameters. a Selection of axial L3 level T1WI slice using coronal T2WI as a reference; b mask generated by ImageJ; c region of interest of muscle segmented manually based on a generated mask; $\mathbf{d}$ example showing the method to measure the lumbar height; e region of interest of SAT segmented manually based on a generated mask; $\mathbf{f}$ region of interest of VAT segmented manually based on the generated mask. SAT: subcutaneous adipose tissue, VAT: visceral adipose tissue 
MRE score $\geq 1$, and no intestinal segment score increased at follow-up.

\section{Statistical analysis}

Statistics were conducted using SPSS 26.0 (IBM Co.) and R software version 3.6.0. Quantitative and categorical variables were described as mean \pm standard deviation (SD) and frequencies (\%), respectively. A paired Student's t-test or a Wilcoxon signed-rank test was performed as appropriate; categorical variables were compared through Chi-squared or Fisher's exact tests. Additionally, we calculated the changes in clinical indicators, body composition parameters, and MRE scores for each longitudinal comparison group at follow-up versus baseline to obtain change values ( $\triangle$ BMI, $\Delta$ CRP, $\Delta$ ESR, $\Delta$ Alb, $\Delta$ Hct, $\Delta$ SMI, $\Delta$ SAI, $\Delta$ VAI, $\triangle \mathrm{VA} / \mathrm{TA}$ index, and $\triangle \mathrm{MRE}$ score). The specific calculation is as follows: $\Delta(i)=(i)$ Value $_{\text {follow-up }}-(i)$ Value $_{\text {baseline }}$. Correlations between clinical and MR changes, height and height $_{\mathrm{L1}-\mathrm{L} 5}$, and standardized indices (SMI and $\mathrm{SMI}_{\text {spinal }}$, SAI and $\mathrm{SAI}_{\text {spinal, }}$ and VAI and $\left.\mathrm{VAI}_{\text {spinal }}\right)$ were evaluated using Spearman/Pearson correlation analysis $(0.3 \leq|r|<0.7$, moderate; $|r|>0.7$, high). Correlation between body composition parameters changes and total MRE score changes were further adjusted with age, sex, follow-up duration and treatment type using multivariate linear regression analysis. Follow-up duration was defined as the interval between two MRE scans per patient. Treatment type was categorized as biologic therapy and non-biologic therapy (other therapies without induction of biological agent). Intraclass correlation coefficient (ICC) or weighted kappa coefficient was used to assess the reliability of body composition area measurements and bowel assessment results between two radiologists; two-tailed $p$-values $<0.05$ were considered significant.

\section{Results}

There were 49 patients enrolled in this study (Fig. 2). Two MRE scans per patient comprised the longitudinal comparison groups and were used to extract body composition parameters further.

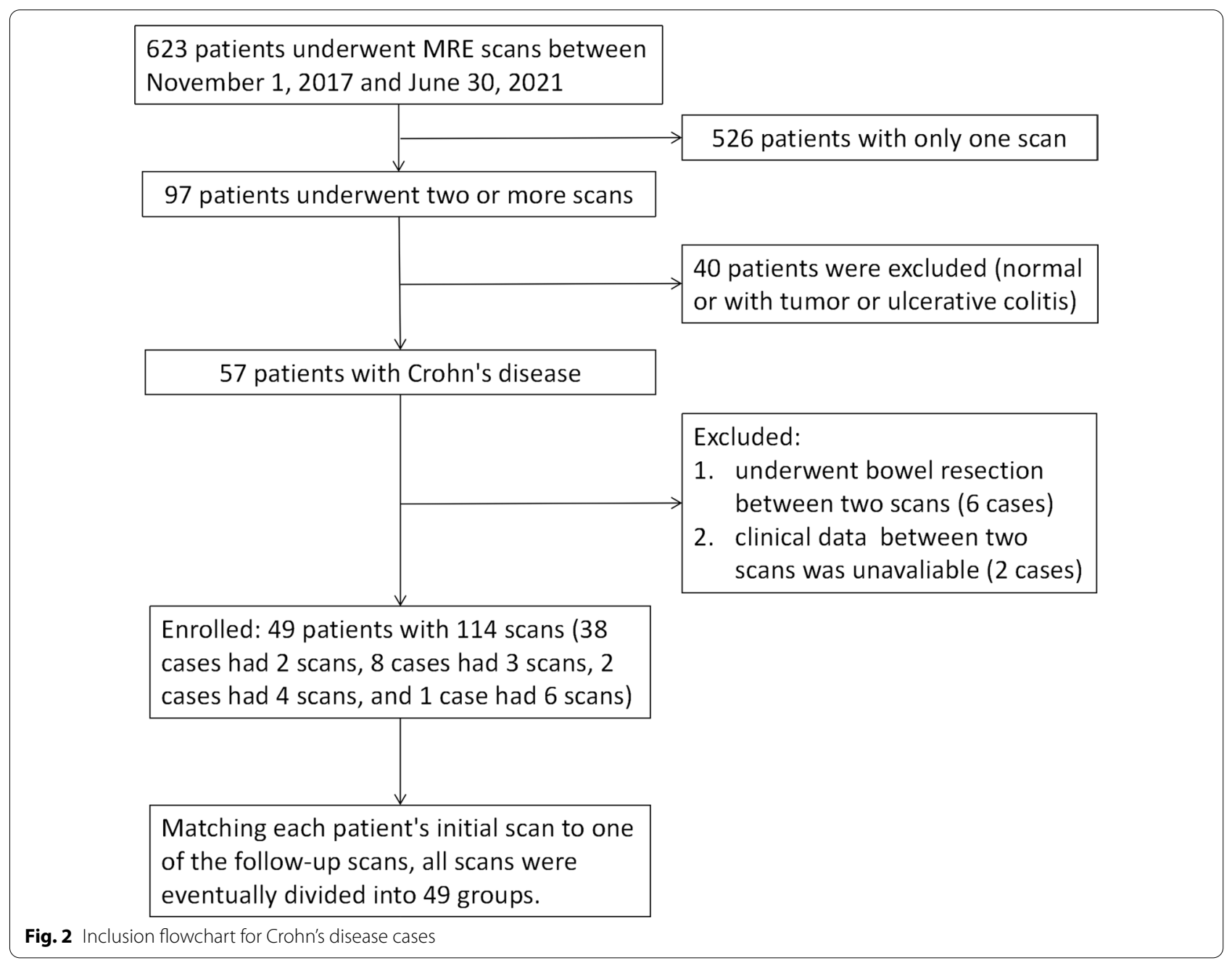


Table 1 Clinical features of all Crohn's disease patients

\begin{tabular}{ll}
\hline Characteristics & Baseline $(\boldsymbol{n}=\mathbf{4 9})$ \\
\hline Age at inclusion, years (mean \pm SD) & $25.82 \pm 9.90$ \\
Gender (male: female) & $3.9: 1$ \\
Active smokers, $n$ (\%) & $10(20.4)$ \\
Scanning interval, months (median, IQR) & $4.5(3.5,7.5)$ \\
Treatment, $n$ (\%) & \\
Biological agents & $38(77.6)$ \\
$\quad$ Infliximab & 29 \\
$\quad$ Adalimumab & 4 \\
$\quad$ Vedolizumab & 3 \\
$\quad$ Ustekinumab & 2 \\
Enteral nutrition & $7(14.3)$ \\
Immunosuppressant + corticosteroid & $3(6.1)$ \\
5-ASA & $1(2.0)$ \\
\hline
\end{tabular}

SD Standard deviation, IQR interquartile range, 5-ASA 5-aminosalicylic acid

\section{Clinical characteristics}

The demographic characteristics of all CD patients are shown in Table 1. Baseline and follow-up clinical information for all patients are shown in Table 2, where baseline information refers to the data obtained when the patients had their first MRE scan at our facility. Patients were predominantly young males, and none indicated a family history of CD. Thirty-eight (77.6\%) patients were treated with biological agents (predominantly infliximab, 29/38 cases), seven with enteral nutrition, three with immunosuppressants (azathioprine), and corticosteroids, and one with 5-ASA. During the follow-up period, the patient's disease site and behaviour did not change significantly. Various laboratory parameters statistically changed, including a significant decrease in CRP and ESR and a significant increase in Alb and Hct $(p<0.001)$. BMI values of all patients increased $\left(18.27 \pm 3.04 \mathrm{~kg} / \mathrm{m}^{2} \mathrm{vs}\right.$. $\left.19.55 \pm 2.66 \mathrm{~kg} / \mathrm{m}^{2} ; p<0.001\right)$, and there was also a significant increase in the proportion of patients within the normal BMI range (36.7\% vs. $57.1 \%)$.

\section{Body composition and intestinal lesion assessment}

By analysing patients' heights and lumbar spine heights in all 98 scans, we found a high correlation between

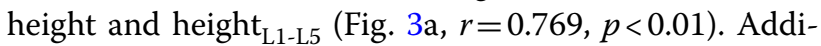
tionally, high correlations were observed between SMI and $\mathrm{SMI}_{\text {spinal }}$, SAI and $\mathrm{SAI}_{\text {spinal }}$, and VAI and $\mathrm{VAI}_{\text {spinal }}$ (Fig. 3b-d, $r=0.952,0.991$ and 0.987 , respectively; $p<0.01)$.

The ICCs for inter-rater reliability in quantitative measurements of skeletal muscle, SAT, and VAT area were $0.982,0.999$, and 0.999 , respectively $(p<0.001)$. For the qualitative assessment of the intestine, the weighted
Table 2 Clinical data of all Crohn's disease patients at initial and follow-up scan

\begin{tabular}{|c|c|c|c|}
\hline Characteristics & Baseline $(n=49)$ & Follow-up $(n=49)$ & $p$-value \\
\hline \multicolumn{4}{|c|}{ Montreal classification } \\
\hline \multicolumn{4}{|c|}{ Age at diagnosis, n (\%) } \\
\hline $\mathrm{A} 1$ & $11(22.4)$ & & \\
\hline$A 2$ & $34(69.4)$ & & \\
\hline A3 & $4(8.2)$ & & \\
\hline Location, n (\%) & & & 0.93 \\
\hline L1 & $17(34.7)$ & $15(30.6)$ & \\
\hline L2 & $1(2.0)$ & $2(4.1)$ & \\
\hline L3 & $30(61.2)$ & $31(63.3)$ & \\
\hline $\mathrm{L} 3+4$ & $1(2.0)$ & $1(2.0)$ & \\
\hline Behaviour, n (\%) & & & 0.93 \\
\hline B1 & $31(63.3)$ & $28(57.1)$ & \\
\hline B2 & $11(22.4)$ & $13(26.5)$ & \\
\hline B3 & $5(10.2)$ & $6(12.2)$ & \\
\hline $\mathrm{B} 2+3$ & $2(4.1)$ & $2(4.1)$ & \\
\hline $\begin{array}{l}\text { Perianal disease, } \\
n(\%)\end{array}$ & $28(57.1)$ & $29(59.2)$ & 1.00 \\
\hline $\begin{array}{l}\mathrm{BMI}, \mathrm{kg} / \mathrm{m}^{2} \\
(\mathrm{mean} \pm \mathrm{SD})\end{array}$ & $18.27 \pm 3.04$ & $19.55 \pm 2.66$ & $<0.001$ \\
\hline $\begin{array}{l}\text { BMI classification, } \\
n(\%)\end{array}$ & & & 0.047 \\
\hline Normal & $18(36.7)$ & $28(57.1)$ & \\
\hline Underweight & $29(59.2)$ & $21(42.9)$ & \\
\hline Overweight & $2(4.1)$ & 0 & \\
\hline \multicolumn{4}{|c|}{ Laboratory findings (mean \pm SD) } \\
\hline $\mathrm{CRP}, \mathrm{mg} / \mathrm{L}(n=46)$ & $35.34 \pm 43.39$ & $11.71 \pm 20.98$ & $<0.001$ \\
\hline $\begin{array}{l}\mathrm{ESR}, \mathrm{mm} / \mathrm{h} \\
(n=48)\end{array}$ & $23.85 \pm 17.97$ & $11.04 \pm 13.51$ & $<0.001$ \\
\hline Alb, g/L $(n=46)$ & $38.43 \pm 5.85$ & $42.19 \pm 5.42$ & $<0.001$ \\
\hline $\mathrm{HCT}, \%(n=47)$ & $34.70 \pm 6.15$ & $38.71 \pm 5.71$ & $<0.001$ \\
\hline
\end{tabular}

$p$ value of variables with great statistical difference between baseline and follow-up is marked in bold

$S D$ Standard deviation, $B M I$ body mass index, $C R P C$-reactive protein, ESR erythrocyte sedimentation rate, Alb albumin, Hct haematocrit

kappa coefficients for each segment were shown in Additional file 1: Table S2, and the total MRE scores obtained were also well reproducible $(\mathrm{ICC}=0.716, p<0.001)$.

\section{Changes in body composition and MRE scores during follow-up}

As shown in Fig. 4a-c, area of skeletal muscle $\left(93.26 \pm 26.15 \mathrm{~cm}^{2}\right.$ vs. $\left.104.50 \pm 27.24 \mathrm{~cm}^{2}\right)$ and SAT $\left(65.88 \pm 48.87 \mathrm{~cm}^{2}\right.$ vs. $\left.78.93 \pm 43.70 \mathrm{~cm}^{2}\right)$ increased significantly during follow-up $(p<0.001$ and $p<0.01$, respectively). Representative images showing the body composition area changes in three patients are illustrated in Fig. 5. Accordingly, indexes including SMI $\left(32.08 \pm 7.71 \mathrm{~cm}^{2} / \mathrm{m}^{2}\right.$ vs. $\left.35.83 \pm 8.05 \mathrm{~cm}^{2} / \mathrm{m}^{2}\right), \mathrm{SMI}_{\text {spinal }}$ $\left(35.41 \pm 8.81 * 10^{2} \mathrm{~cm}^{2} / \mathrm{m}^{2}\right.$ vs. $39.51 \pm 9.14 * 10^{2} \mathrm{~cm}^{2} /$ 

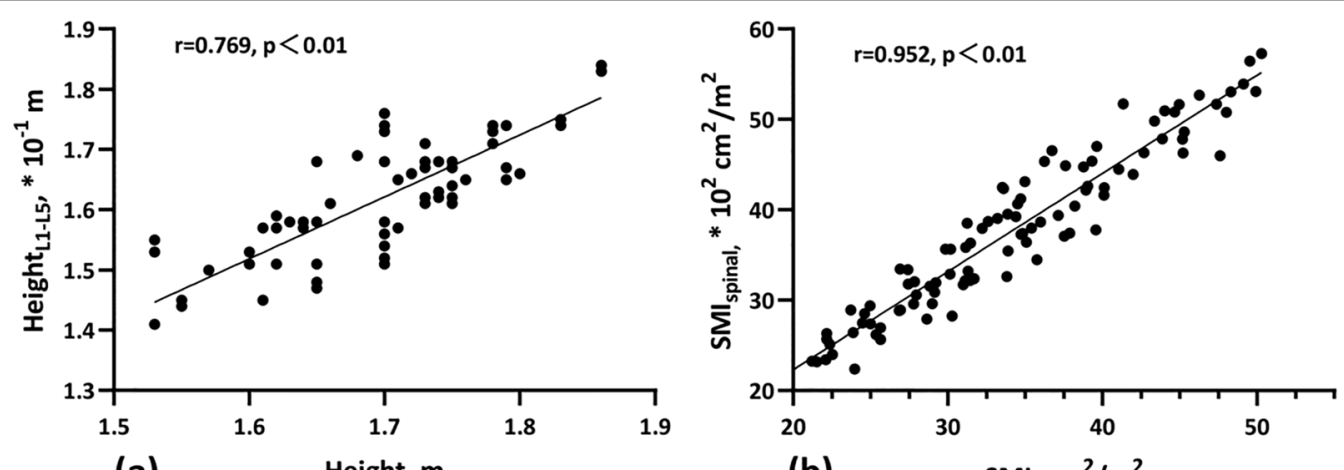

(a)

(b)

$\mathrm{SMI}, \mathrm{cm}^{2} / \mathrm{m}^{2}$

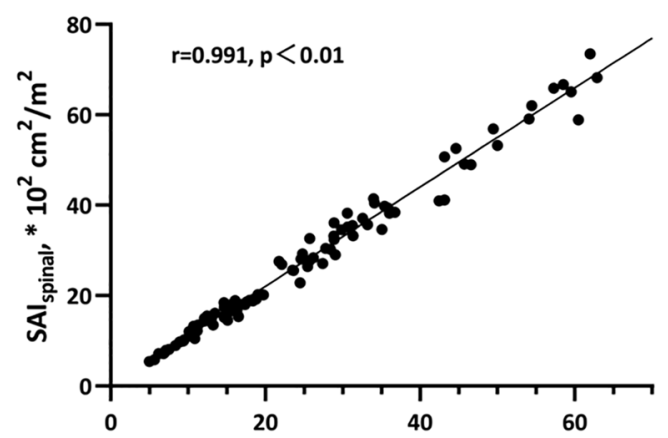

(c)

$\mathrm{SAl}, \mathrm{cm}^{2} / \mathrm{m}^{2}$

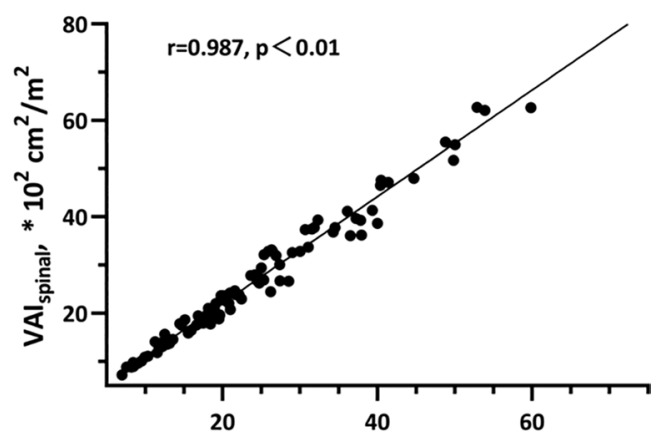

(d)

$\mathrm{VAl}, \mathrm{cm}^{2} / \mathrm{m}^{2}$

Fig. 3 Scatter plots showing the significant positive correlations between height and the height of the 1 st to 5 th lumbar vertebrae (a), as well as between the standardized indices obtained via two different methods (b-d)

$\left.\mathrm{m}^{2}\right)$, SAI $\left(23.10 \pm 16.87 \mathrm{~cm}^{2} / \mathrm{m}^{2}\right.$ vs. $27.65 \pm 15.75 \mathrm{~cm}^{2} /$ $\left.\mathrm{m}^{2}\right)$, and $\operatorname{SAI}_{\text {spinal }}\left(25.50 \pm 18.58 * 10^{2} \mathrm{~cm}^{2} / \mathrm{m}^{2}\right.$ vs. $\left.30.46 \pm 17.51 * 10^{2} \mathrm{~cm}^{2} / \mathrm{m}^{2}\right)$ showed the same increasing trend during follow-up $(p<0.001$ for SMI and $\mathrm{SMI}_{\text {spinal }}$, and $p<0.01$ for SAI and $\mathrm{SAI}_{\text {spinal }}$, respectively). No significant changes were observed in VAT area $\left(65.88 \pm 31.37 \mathrm{~cm}^{2}\right.$ vs. $\left.75.10 \pm 41.49 \mathrm{~cm}^{2}\right)$ and VAI $\left(23.01 \pm 10.85 \mathrm{~cm}^{2} / \mathrm{m}^{2}\right.$ vs. $26.00 \pm 13.92 \mathrm{~cm}^{2} /$ $\left.\mathrm{m}^{2}\right)(p>0.05)$. VAI $\mathrm{I}_{\text {spinal }}\left(25.39 \pm 12.00 * 10^{2} \mathrm{~cm}^{2} / \mathrm{m}^{2}\right.$ vs. $\left.28.72 \pm 15.66 * 10^{2} \mathrm{~cm}^{2} / \mathrm{m}^{2}\right)$ showed a slightly increase during follow-up $(p=0.04)$. On the contrary, VA/TA index decreased significantly during the same process $(52.69 \pm 10.66 \%$ vs. $49.18 \pm 10.80 \%, p<0.001)$ (Fig. $4 d$ ). Sarcopenia presented in all patients at the time of the first MRE scan and presented in 37 males and 7 females after follow-up ( $p=0.77)$. Additionally, the small bowel, colorectum and total MRE scores all decreased significantly during the follow-up period (Fig. 4e).

\section{Correlation among clinical indicators changes, MRE score} changes, and body composition changes during follow-up The correlation coefficients for changes in clinical indicators, MRE score, and body composition parameters are shown in Additional file 1: Table S3. We found that $\triangle \mathrm{BMI}$ was moderately to highly positively correlated with $\Delta \mathrm{SMI}$, $\Delta$ SAI, and $\Delta$ VAI $(r=0.593,0.779$, and 0.568 , respectively; $p<0.01)$, and moderately negatively correlated with the $\Delta$ VA/TA index $(r=-0.422 ; p<0.01)$ (Fig. 6a). The correlation between $\triangle \mathrm{CRP}$ and body composition parameters was only reflected in the $\Delta \mathrm{VA} / \mathrm{TA}$ index $(r=0.355$, $p<0.05$ ) (Fig. 6b). $\Delta$ ESR was moderately negatively correlated with $\triangle \mathrm{SMI}(r=-0.481, p<0.01)$ but positively correlated with $\triangle \mathrm{VA} / \mathrm{TA}$ index $(r=0.332, p<0.05)$ (Fig. 6c). The $\triangle$ total MRE score showed a negative correlation with both $\Delta$ SMI and $\Delta$ SAI $(r=-0.408, p<0.01 ;-0.320$, $p<0.05)$ and a positive correlation with the $\Delta \mathrm{VA} / \mathrm{TA}$ index $(r=0.479, p<0.01) \quad$ (Fig. 6d). The correlation between $\triangle$ VAI and $\Delta$ total MRE score was not observed $(r=-0.020, p>0.05)$. Additionally, results showed that $\Delta$ SMI was positively correlated with both $\Delta \mathrm{Alb}$ and $\Delta \mathrm{Hct}$ $(r=0.450$ and 0.422 , respectively $p<0.01)$ and negatively correlated with $\triangle \mathrm{VA} / \mathrm{TA}$ index $(r=-0.339, p<0.05)$. Furthermore, we found that the differences in SMI, SAI, and VAI during follow-up were mainly in patients using biological agents, while the differences in VA/TA index were significant for all therapy methods. Additionally, the patients' intestinal improvement was not affected by the treatment modality (Table 3 ). When adjusted by variates, 


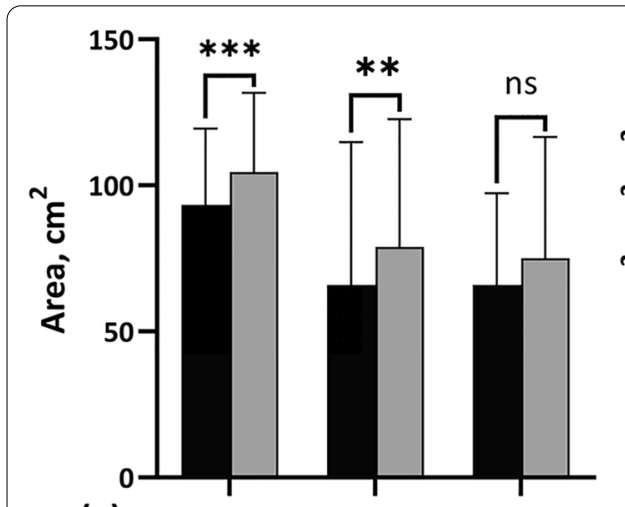

(a) Skeletal muscle SAT
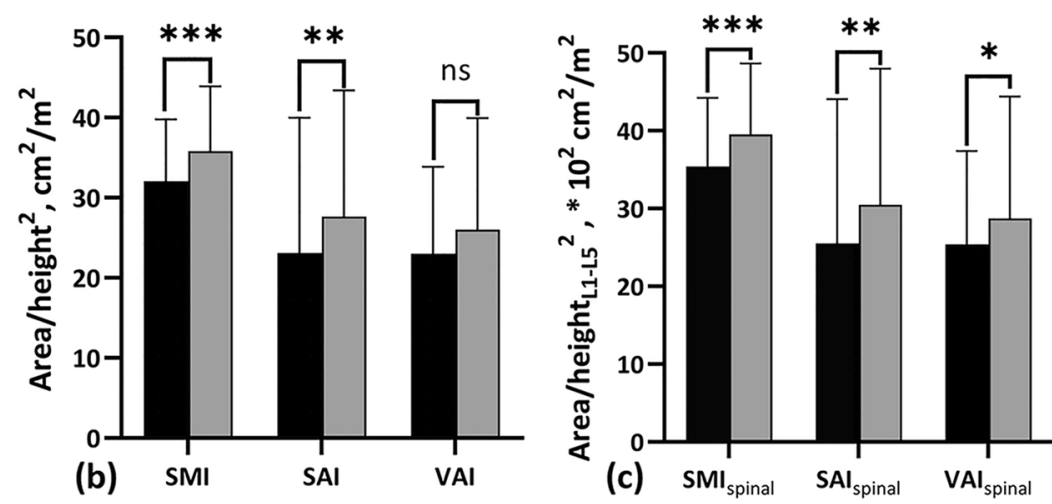

(c) $\mathrm{SMI}_{\text {spinal }} \quad \mathrm{SAI}_{\text {spinal }} \quad \mathrm{VAI}_{\text {spinal }}$

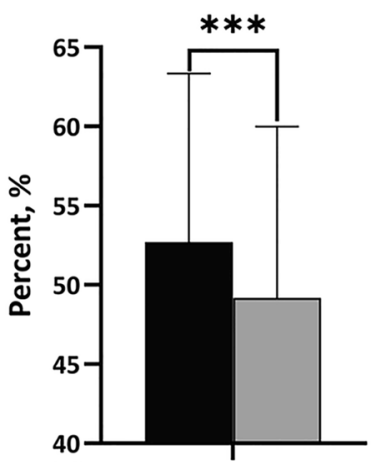

(d)

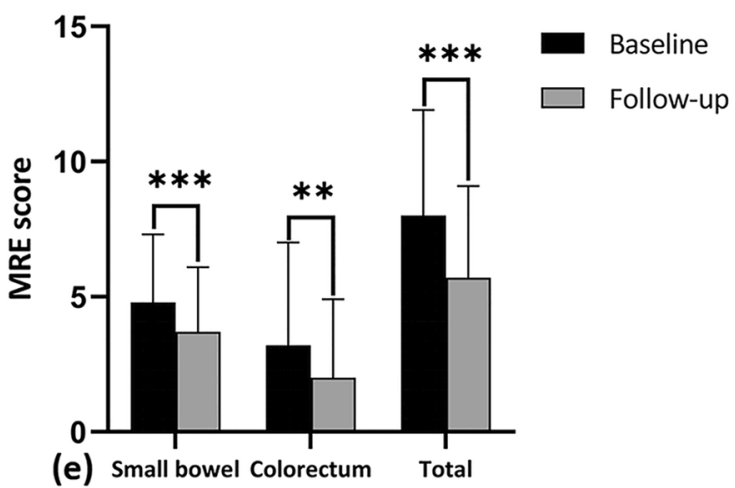

Fig. 4 Bar graphs of body composition parameters and MRE scores for all cases. Black bars represent baseline data, and grey bars represent follow-up data. a Area of skeletal muscle, SAT, and VAT at baseline and follow up; b Value of SMI, SAI, and VAI at baseline and follow up; c Value of SMIspinal, SAlspinal, and VAlspinal at baseline and follow up; $\mathbf{d}$ Value of VA/TA index at baseline and follow up; e MRE scores of small bowel and colorectum at baseline and follow up. ${ }^{* * *}: p<0.001,{ }^{* *}: p<0.01,{ }^{*}: p<0.05, \mathrm{~ns}: p>0.05$ (not significant); SAT: subcutaneous adipose tissue,

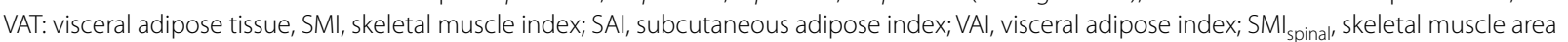

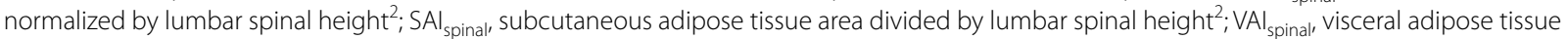
area divided by lumbar spinal height ${ }^{2}$; ns, no significant difference; VA/TA index, VAT to total adipose tissue ratio

including sex, age, follow-up duration, and treatment type, $\triangle \mathrm{VA} / \mathrm{TA}$ index was still strongly associated with the $\Delta$ total MRE score (Table 4).

\section{Discussion}

This retrospective, longitudinal study explored changes in clinical indicators, disease activity, and body composition parameters in $\mathrm{CD}$ patients during follow-up. In the present study, the change in VAT percentage (VA/TA index) and SMI during treatment in CD patients can be used as feasible indicators to monitor overall nutritional and inflammatory status, since $\triangle \mathrm{VA} / \mathrm{TA}$ index was correlated well with changes in CRP, ESR and MRE activity score, while $\triangle$ SMI correlated linearly with changes in BMI, Alb, Hct, and MRE activity score during follow-up.

After treatment, a significant increase in skeletal muscle, SAT, and VAT area was found, while an unexpected decrease in the relative visceral fat content (VA/
TA index) was observed. The changes in skeletal muscle, SAT, and VAT during treatment were positively correlated with the evolution of BMI, whereas the VA/TA index was negatively correlated. Drug therapy-induced weight gain in CD patients has been reported [5]. A prospective study found that anti-TNF therapy caused a rapid and uniform gain in abdominal fat; i.e., the increase occurred uniformly in both SAT and VAT [21]. This is somewhat similar to findings in the present study; however, they did not observe a link between these indicators and trends in BMI nor conduct further analysis targeting the relative VAT content.

Adipose tissue has been described as an endocrine organ that profoundly impacts metabolism [22]. In humans, SAT and VAT have been shown to have significantly different gene expression patterns [23, 24]. VAT inflammation has long been recognized as important in the pathogenesis of $\mathrm{CD}$; its encapsulation of diseased 


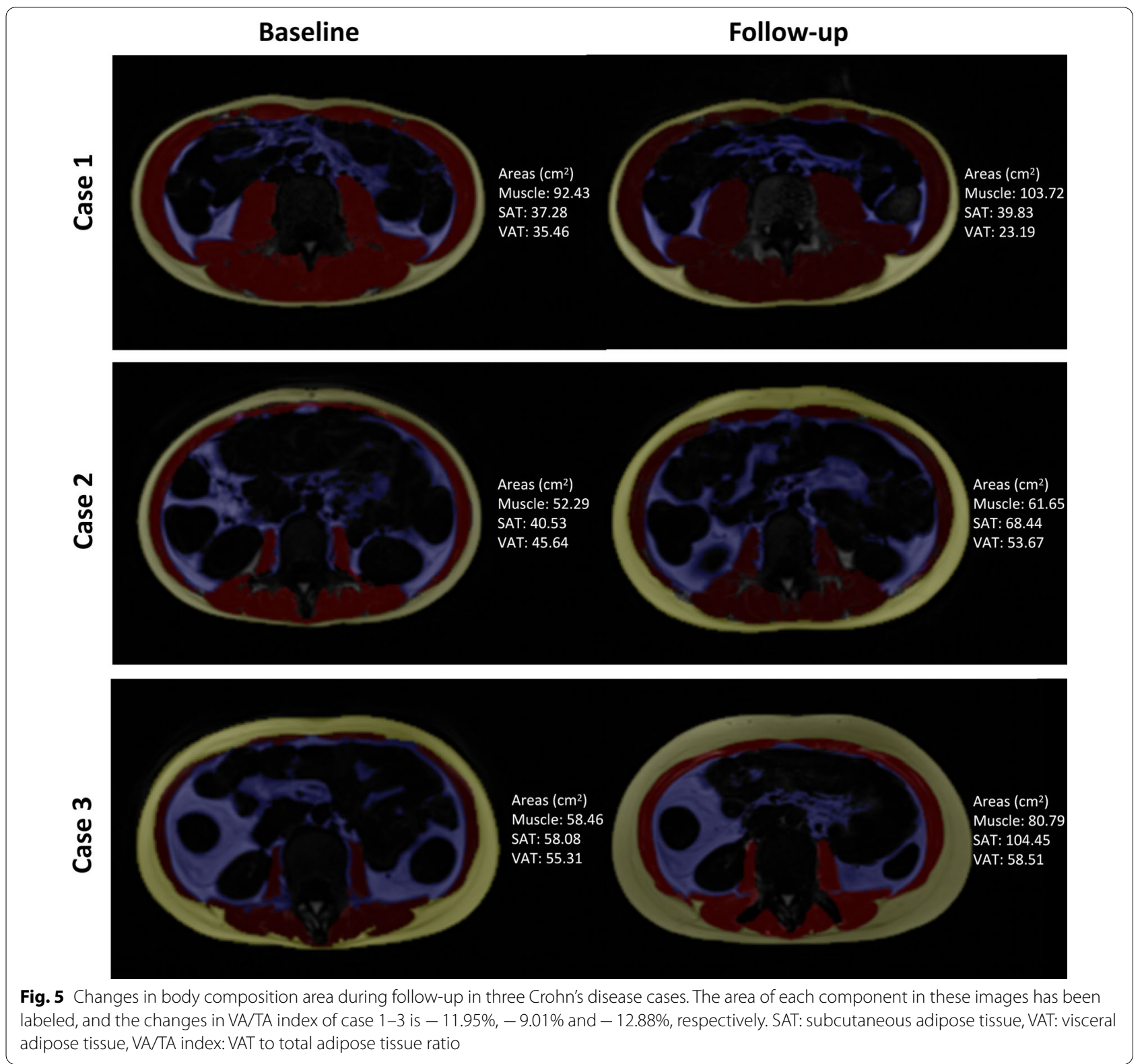

Table 3 Effect of therapy on body composition parameters and intestinal improvement

\begin{tabular}{llllcc}
\hline & $\begin{array}{l}\text { Biological agents } \\
(\boldsymbol{n}=\mathbf{3 8})\end{array}$ & $\begin{array}{l}\text { Non-biological agents } \\
(\boldsymbol{n}=\mathbf{1 1})\end{array}$ & $\boldsymbol{P 1}$ & $\boldsymbol{P 2}$ & $\boldsymbol{P 3}$ \\
\hline$\triangle \mathrm{SMI}, \mathrm{cm}^{2} / \mathrm{m}^{2}$ & $4.38 \pm 4.61$ & $1.62 \pm 4.46$ & $<\mathbf{0 . 0 0 1}$ & 0.26 & $\mathbf{0 . 0 4 9 9}$ \\
$\triangle \mathrm{SAl}, \mathrm{cm}^{2} / \mathrm{m}^{2}$ & $4.51 \pm 8.07$ & $4.68 \pm 17.13$ & $<\mathbf{0 . 0 0 1}$ & 0.39 & 0.93 \\
$\triangle \mathrm{VAl}, \mathrm{cm}^{2} / \mathrm{m}^{2}$ & $3.75 \pm 10.47$ & $0.37 \pm 11.22$ & $\mathbf{0 . 0 1 7}$ & 0.92 & 0.61 \\
$\triangle \mathrm{VA} / \mathrm{TA}$ index, $\%$ & $-3.22 \pm 7.23$ & $-4.51 \pm 4.63$ & $\mathbf{0 . 0 0 9}$ & $\mathbf{0 . 0 0 9}$ & 0.58 \\
Intestinal improvement, $n$ & 24 & 6 & - & - & 0.73 \\
\hline
\end{tabular}

$p$ values below 0.05 are marked in bold

$S M I$ Skeletal muscle index, $S A I$ subcutaneous adipose tissue index, VAI visceral adipose tissue index, VA/TA area of visceral fat/area of subcutaneous fat; $P 1$ intra-group differences between baseline and follow-up values in patients group with biologic therapy type; $P 2$ intra-group differences between baseline and follow-up values in patients group with non-biologic therapy type; $P 3$ inter-group differences evaluated using the independent samples $t$-test or Mann-Whitney $U$ test, and the $X^{2}$ test 


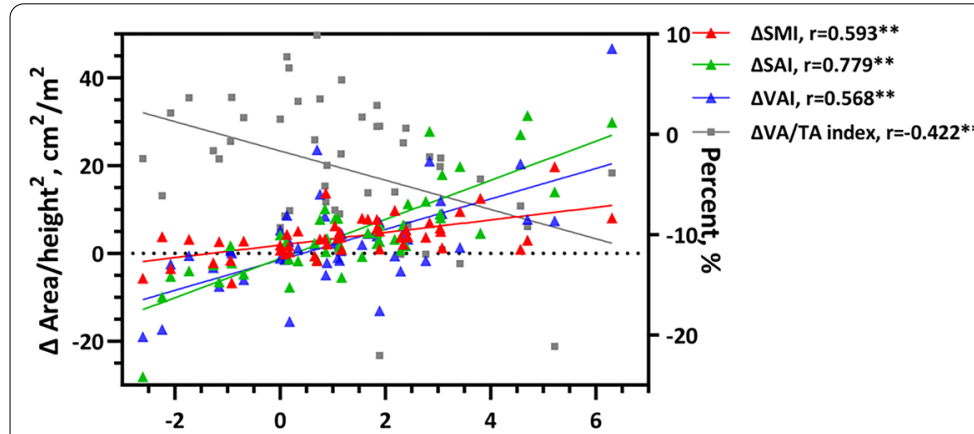

(a)

$\Delta B M I, \mathrm{~kg} / \mathrm{m}^{2}$

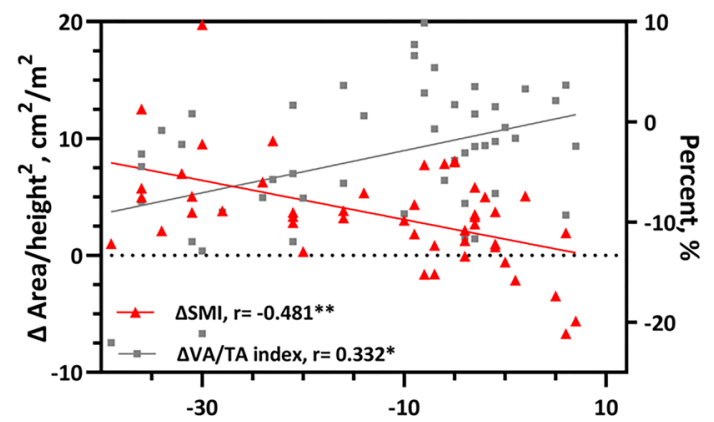

(c)

$\Delta$ ESR, $\mathrm{mm} / \mathrm{hr}$
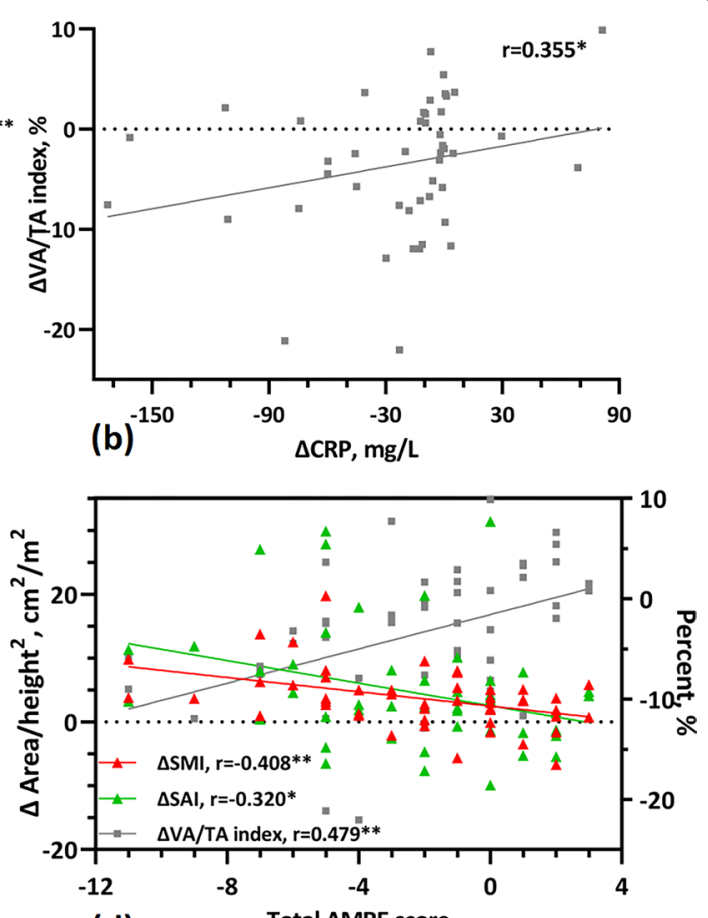

(d)

Fig. 6 Scatter plots showing the correlation between changes in clinical indicators, MRE score, and body composition parameters during follow-up. a Correlation between changes of body composition parameters ( $\triangle \mathrm{SMI}, \triangle \mathrm{SAl}, \triangle \mathrm{VAl}$, and $\triangle \mathrm{VA} / T \mathrm{TA}$ ) and $\mathrm{BMI}$; $\mathbf{b}$ Correlation between $\triangle \mathrm{VA} / T \mathrm{~A}$ and $\triangle \mathrm{CRP}$; c Correlation between changes of body composition parameters ( $\triangle \mathrm{SMI}$ and $\triangle \mathrm{VA} / \mathrm{TA}$ ) and ESR; $\mathbf{d}$ Correlation between changes of body composition parameters ( $\triangle S \mathrm{SMI}, \triangle \mathrm{SAl}$, and $\triangle \mathrm{VA} / \mathrm{TA}$ ) and total MRE score. ${ }^{* *}: p<0.01,{ }^{*}: p<0.05 ; \mathrm{BMI}$, body mass index; CRP, C-reactive protein; ESR, Erythrocyte sedimentation rate; SMI, skeletal muscle index; SAI, subcutaneous adipose index; VAI, visceral adipose index, VA/TA index: VAT to total adipose tissue ratio; $\Delta(i)=(i) V_{\text {alue }}$ follow-up $-(i) V_{\text {alue }}$ baseline, $i=B M I, S M I, S A I, V A I, V A / T A, C R P, E S R$, MRE score

intestinal segments to form the so-called "creeping fat" is an important source of pro-inflammatory cytokines (e.g., IL-6 and TNF- $\alpha$ ) [25-27]. Contrary to the negative effects of abnormal VAT accumulation, several studies have confirmed SAT to benefit metabolism [28-30]. The present study showed that the $\triangle \mathrm{VA} / \mathrm{TA}$ index was positively correlated with the trends in CRP, ESR, and total MRE score $(r=0.355, p<0.05 ; r=0.332, p<0.05$; $r=0.479, p<0.01$ ), while $\Delta$ SAI was negatively correlated with $\Delta$ total MRE score $(r=-0.320, p<0.05)$. Labarthe et al. [13] also observed that active/severe CD patients had a higher VA/TA index and lower SAI than inactive patients, similar to the present results. Still, we focus more on analysing changes in disease over treatment, which is more applicable to monitoring during follow-up.

Table 4 Correlation between body composition parameters changes and $\triangle$ total MRE scores with adjustment of multi-variables

\begin{tabular}{|c|c|c|c|c|c|c|c|c|c|}
\hline \multirow[t]{2}{*}{ Variables } & \multicolumn{3}{|l|}{$\Delta \mathrm{VA} / \mathrm{TA}$} & \multicolumn{3}{|l|}{$\Delta \mathrm{SMI}$} & \multicolumn{3}{|l|}{$\Delta \mathrm{SAl}$} \\
\hline & Coefficient & SD & $p$ value & Coefficient & SD & $p$ value & Coefficient & SD & $p$ value \\
\hline Intercept & -7.69 & 3.13 & 0.017 & 2.10 & 2.31 & 0.369 & 3.19 & 5.06 & 0.532 \\
\hline Biologic therapy & 2.15 & 2.06 & 0.302 & 1.90 & 1.52 & 0.219 & -1.49 & 3.33 & 0.656 \\
\hline Follow-up duration & 0.16 & 0.16 & 0.308 & 0.02 & 0.12 & 0.868 & 0.68 & 0.25 & 0.010 \\
\hline Age & 0.15 & 0.09 & 0.092 & -0.03 & 0.06 & 0.632 & -0.17 & 0.14 & 0.226 \\
\hline Sex (female) & -2.48 & 2.10 & 0.245 & -1.31 & 1.55 & 0.403 & 5.46 & 3.41 & 0.117 \\
\hline$\Delta$ total MRE scores & 0.84 & 0.25 & 0.001 & -0.50 & 0.19 & 0.010 & -0.67 & 0.41 & 0.109 \\
\hline
\end{tabular}

$p$ values below 0.05 are marked in bold

MRE Magnetic resonance enterography, SMI skeletal muscle index, SAI subcutaneous adipose tissue index, VA/TA area of visceral fat/area of subcutaneous fat 
Therefore, changes in the two fat parameters could reflect changes in the inflammatory state.

Although data indicates that the prevalence of overweight and obesity is increasing in the $C D$ population and that increased VAT caused by obesity promotes disease progression [31], in patients with a low BMI, hypertrophic mesenteric adipose tissue is observed early in the onset of the disease [32]. Moreover, even though most CD patients exhibited a normal or even low BMI, they still had a higher percentage of VAT when compared with healthy subjects [33]. In the present study, twentynine patients $(59.2 \%)$ had low baseline BMIs. After treatment, their overall BMI had improved, while a significant reduction in VAT percentage occurred at the same time. Therefore, we believe that BMI may be misleading and does not provide a good indication of changes in abnormal VAT for accurate determination of disease progression status.

Monitoring the nutritional status of $\mathrm{CD}$ patients during treatment is important for their quality of life [34]. Skeletal muscle content and density are indexes to assess patients' nutritional condition in previous studies, and sarcopenia is often used to describe a combined loss of muscle function and mass. Wiese et al. [35] presented that infliximab reversed poor nutritional status in $C D$, while Subramaniam et al. [36] showed increased muscle mass in patients treated with anti-TNF. All patients in this study suffered sarcopenia at their inclusion. After subsequent treatment, their nutritional status showed an overall improvement, as evidenced by an increase in SMI and a decrease in patients who suffered sarcopenia. A consistent finding is that the increase in SMI was paralleled by improvements in BMI and laboratory nutritional index like Alb and Hct, as $\triangle$ SMI was positively correlated with trends in Alb and Hct $(r=0.450$ and 0.422, respectively, $p<0.01$ ). Additionally, the increase of SMI and decrease of VA/TA index were strongly associated with improvement in disease activity reflected by the decrease of total MRE score after adjustment of multiple variables. An explanation for this is that CD patients often have nutrient absorption issues, mainly reflected in reduced fat and protein content, leading to amino acid deficiency and hypoproteinaemia [37]. Hypoproteinaemia occurs not only due to decreased synthesis but also increased catabolism during inflammation [3]. Therefore, changes in the nutritional status and disease activity of $\mathrm{CD}$ patients during treatment can be well detected by body composition parameters, including SMI and VA/TA index.

In this study, a method for area normalization using height $_{\text {L1-L5 }}$ instead of height was proposed. We found a strong correlation between the standardized indices of skeletal muscle, SAT, and VAT obtained by the two routes $(r=0.952,0.991$, and 0.987 , respectively; $p<0.01$ ). Height $_{\text {L1-L5 }}$ is predominant in conditions when accurate standing height data was unavailable, like emergency admission. If height can be replaced by height ${ }_{\mathrm{L} 1-\mathrm{L} 5}$ measured directly on the images, patients excluded due to lack of height data can be decreased and thus increase the statistical power of similar retrospective studies. Further large-scale prospective research was needed to facilitate the application of height $\mathrm{L} 1-\mathrm{L} 5$ in body composition analysis.

This study has some limitations. First, due to its retrospective design, some clinical information like endoscopic data was not available at the time of each MRE scan. Correspondingly, we evaluated the disease activity of each patient by scoring the intestinal lesions in each MRE image. Information was also incomplete for calculating the Crohn's disease activity index in most $\mathrm{CD}$ patients at their admission. Further study is needed for verifying the significant changes of SMI and VA/TA with inflammatory status reflected by endoscopic data or Crohn's disease activity index. Second, selection bias may exist due to the relatively small sample size and single centre design. Finally, although the current semiautomated segmentation method can obtain accurate body composition parameters, it is still a time-consuming process. To promote clinical practicability, the same precise automated segmentation methods should be developed in future works.

In conclusion, body composition analysis based on MRI is reproducible; the information obtained, especially the SMI and VA/TA index, can be used to monitor the nutritional and inflammatory status of $\mathrm{CD}$ patients during follow-up.

\section{Abbreviations}

Alb: Albumin; BMl: Body mass index; CD: Crohn's disease; CRP: C-reactive protein; ESR: Erythrocyte sedimentation rate; Hct: Haematocrit; MRE: Magnetic resonance enterography; SAI: Subcutaneous adipose index; SAT: Subcutaneous adipose tissue; SMI: Skeletal muscle index; VAl: Visceral adipose index; VAT: Visceral adipose tissue.

\section{Supplementary Information}

The online version contains supplementary material available at https://doi. org/10.1186/s13244-021-01121-3.

Additional file 1. Table S1. MRE protocol. Table S2. Kappa coefficients for each intestinal segment in the MRE assessment. Table S3. Correlation between changes in body composition and clinical indicators during treatment (Spearman coefficient or Pearson coefficient).

\section{Acknowledgements}

We are grateful to the gastroenterologists (Fang Xiao; Jian Han; Yu Chen) and pathologist (Jun Wang; Chao Zhang) of the inflammatory bowel disease multidisciplinary team at Tongji Hospital for their assistance in the diagnosis of $C D$ patients. 


\section{Authors' contributions}

YS: Conceptualization, Methodology, and Funding acquisition; ZZ and ZX: Methodology, Formal analysis, Data Curation, Writing-Original Draft, and Visualization; ZL: Writing-Review and Editing and Funding acquisition; $\mathrm{XH}$ : Investigation and Funding acquisition; DH: Supervision and Funding acquisition. All authors read and approved the final manuscript.

\section{Funding}

This work is supported by the grants from National Natural Science Foundation of China (NSFC) Nos. 82071890, 81801695, 81771801, 82071889 and 81571642 .

\section{Availability of data and materials}

The datasets used or analyzed during the current study are available from the corresponding author on reasonable request.

\section{Declarations}

\section{Ethics approval and consent to participate}

This retrospective study was approved by the Ethics Committee of Tongji Hospital and the informed consent of all patients was waived.

\section{Consent for publication}

This manuscript is approved by all patients for publication.

\section{Competing interests}

All authors declare no conflict of interest in present study.

\section{Author details}

'Department of Radiology, Tongji Hospital, Tongji Medical College, Huazhong University of Science and Technology, 1095 Jiefang Avenue, Qiaokou District, Wuhan 430030, Hubei, China. ${ }^{2}$ Biomedical Engineering Department, College of Life Sciences and Technology, Huazhong University of Science and Technology, Wuhan, China.

\section{Received: 18 August 2021 Accepted: 1 November 2021}

Published online: 04 December 2021

\section{References}

1. Balestrieri P, Ribolsi M, Guarino MPL, Emerenziani S, Altomare A, Cicala M (2020) Nutritional aspects in inflammatory bowel diseases. Nutrients $12: 372$

2. Takaoka A, Sasaki M, Nakanishi N et al (2017) Nutritional screening and clinical outcome in hospitalized patients with Crohn's disease. Ann Nutr Metab 71:266-272

3. Stokes MA (1992) Crohn's disease and nutrition. Br J Surg 79:391-394

4. Grillot J, D'Engremont C, Parmentier AL et al (2020) Sarcopenia and visceral obesity assessed by computed tomography are associated with adverse outcomes in patients with Crohn's disease. Clin Nutr 39:3024-3030

5. Christian KE, Russman KM, Rajan DP, Barr EA, Cross RK (2020) Gender differences and other factors associated with weight gain following initiation of infliximab: a post hoc analysis of clinical trials. Inflamm Bowel Dis 26:125-131

6. Grossberg AJ, Chamchod S, Fuller CD et al (2016) Association of body composition with survival and locoregional control of radiotherapytreated head and neck squamous cell carcinoma. JAMA Oncol 2:782-789

7. Weston AD, Korfiatis P, Kline TL et al (2019) Automated abdominal segmentation of CT scans for body composition analysis using deep learning. Radiology 290:669-679

8. Huber FA, Chaitanya K, Gross N et al (2021) Whole-body composition profiling using a deep learning algorithm: influence of different acquisition parameters on algorithm performance and robustness. Invest Radiol. https://doi.org/10.1097/RLI.0000000000000799

9. Seidell JC, Bakker CJ, van der Kooy K (1990) Imaging techniques for measuring adipose-tissue distribution - a comparison between computed tomography and 1.5-T magnetic resonance. Am J Clin Nutr 51:953-957
10. Baum T, Cordes C, Dieckmeyer M et al (2016) MR-based assessment of body fat distribution and characteristics. Eur J Radiol 85:1512-1518

11. Rimola J, Rodriguez S, García-Bosch O et al (2009) Magnetic resonance for assessment of disease activity and severity in ileocolonic Crohn's disease. Gut 58:1113-1120

12. Büning $C$, von Kraft C, Hermsdorf M et al (2015) Visceral adipose tissue in patients with Crohn's disease correlates with disease activity, inflammatory markers, and outcome. Inflamm Bowel Dis 21:2590-2597

13. Labarthe $G$, Dolores M, Verdalle-Cazes M et al (2020) Magnetic resonance imaging assessment of body composition parameters in Crohn's disease. Dig Liver Dis 52:878-884

14. Maaser C, Sturm A, Vavricka SR et al (2019) ECCO-ESGAR guideline for diagnostic assessment in IBD Part 1: initial diagnosis, monitoring of known IBD, detection of complications. J Crohns Colitis 13:144-164

15. Satsangi J, Silverberg MS, Vermeire S, Colombel JF (2006) The Montreal classification of inflammatory bowel disease: controversies, consensus, and implications. Gut 55:749-753

16. WHO Expert Consultation (2004) Appropriate body-mass index for Asian populations and its implications for policy and intervention strategies. Lancet 363:157-163

17. Taylor SA, Avni F, Cronin CG et al (2017) The first joint ESGAR/ESPR consensus statement on the technical performance of cross-sectional small bowel and colonic imaging. Eur Radiol 27:2570-2582

18. Gomez-Perez S, McKeever L, Sheean P (2020) Tutorial: a step-by-step guide (version 2.0) for measuring abdominal circumference and skeletal muscle from a single cross-sectional computed-tomography image using the national institutes of health image. J Parenter Enteral Nut 44:419-424

19. Zhang TH, Ding C, Xie TB et al (2017) Skeletal muscle depletion correlates with disease activity in ulcerative colitis and is reversed after colectomy. Clin Nutr 36:1586-1592

20. Kitazume Y, Fujioka T, Takenaka K et al (2019) Crohn disease: a 5-point MR enterocolonography classification using enteroscopic findings. AJR Am J Roentgenol 212:67-76

21. Parmentier-Decrucq E, Duhamel A, Ernst O et al (2009) Effects of infliximab therapy on abdominal fat and metabolic profile in patients with Crohn's disease. Inflamm Bowel Dis 15:1476-1484

22. Pantanetti P, Garrapa GGM, Mantero F, Boscaro M, Faloia E, Venarucci D (2004) Adipose tissue as an endocrine organ? A review of recent data related to cardiovascular complications of endocrine dysfunctions. Clin Exp Hypertens 26:387-398

23. Lefebvre AM, Laville M, Vega N et al (1998) Depot-specific differences in adipose tissue gene expression in lean and obese subjects. Diabetes 47:98-103

24. Tchkonia T, Lenburg $M$, Thomou T et al (2007) Identification of depotspecific human fat cell progenitors through distinct expression profiles and developmental gene patterns. Am J Physiol Endocrinol Metab 292:E298-307

25. Ghigliotti G, Barisione C, Garibaldi S et al (2014) Adipose tissue immune response: novel triggers and consequences for chronic inflammatory conditions. Inflammation 37:1337-1353

26. Drouet M, Dubuquoy L, Desreumaux P, Bertin B (2012) Visceral fat and gut inflammation. Nutrition 28:113-117

27. Paeschke A, Erben U, Kredel LI, Kühl AA, Siegmund B (2017) Role of visceral fat in colonic inflammation: from Crohn's disease to diverticulitis. Curr Opin Gastroenterol 33:53-58

28. Gavrilova O, Marcus-Samuels B, Graham D et al (2000) Surgical implantation of adipose tissue reverses diabetes in lipoatrophic mice. J Clin Invest 105:271-278

29. Misra A, Garg A, Abate N, Peshock RM, Stray-Gundersen J, Grundy SM (1997) Relationship of anterior and posterior subcutaneous abdominal fat to insulin sensitivity in nondiabetic men. Obes Res 5:93-99

30. Tran TT, Yamamoto Y, Gesta S, Kahn CR (2008) Beneficial effects of subcutaneous fat transplantation on metabolism. Cell Metab 7:410-420

31. Moran GW, Dubeau MF, Kaplan GG, Panaccione R, Ghosh S (2013) The increasing weight of Crohn's disease subjects in clinical trials: a hypothesis-generatings time-trend analysis. Inflamm Bowel Dis 19:2949-2956

32. Li Y, Zhu W, Zuo L, Shen B (2016) The role of the mesentery in Crohn's disease: the contributions of nerves, vessels, lymphatics, and fat to the pathogenesis and disease course. Inflamm Bowel Dis 22:1483-1495

33. Desreumaux P, Ernst O, Geboes K et al (1999) Inflammatory alterations in mesenteric adipose tissue in Crohn's disease. Gastroenterology 117:73-81 
34. Werkstetter KJ, Ullrich J, Schatz SB, Prell C, Koletzko B, Koletzko S (2012) Lean body mass, physical activity and quality of life in paediatric patients with inflammatory bowel disease and in healthy controls. J Crohns Colitis 6:665-673

35. Wiese D, Lashner B, Seidner D (2008) Measurement of nutrition status in Crohn's disease patients receiving infliximab therapy. Nutr Clin Pract 23:551-556

36. Subramaniam K, Fallon K, Ruut T et al (2015) Infliximab reverses inflammatory muscle wasting (sarcopenia) in Crohn's disease. Aliment Pharmacol Ther 41:419-428

37. Harries AD, Heatley RV (1983) Nutritional disturbances in Crohn's disease. Postgrad Med J 59:690-697

\section{Publisher's Note}

Springer Nature remains neutral with regard to jurisdictional claims in published maps and institutional affiliations.

\section{Submit your manuscript to a SpringerOpen ${ }^{\circ}$ journal and benefit from:}

- Convenient online submission

- Rigorous peer review

- Open access: articles freely available online

- High visibility within the field

- Retaining the copyright to your article

Submit your next manuscript at $\boldsymbol{\nabla}$ springeropen.com 